\title{
Invasion with Toxoplasma gondii can promote pneumocystis pneumonia in individuals with HIVIAIDS
}

\author{
Ihor H. Hryzhak (D)
}

Ivano-Frankivsk National Medical University, Ivano-Frankivsk, Ukraine and Ivano-Frankivsk Regional Center for AIDS Prevention and Fight, Ivano-Frankivsk, Ukraine

\begin{abstract}
Pulmonary pathology is common in HIV-infected individuals, but the possible role of the parasitic protist Toxoplasma gondii (Nicolle et Manceaux, 1908) is not completely known. The present account reports result of a retrospective cohort study. Medical cards of 907 HIV-positive people, which included 120 deceased patients, were analysed. During a three-year follow-up, the pulmonary pathology was diagnosed in 306 patients $(33.7 \pm 1.6 \%)$ : pneumocystis pneumonia in $124(13.7 \pm 1.1 \%)$, primary pulmonary tuberculosis in $113(12.5 \pm 1.1 \%)$, bacterial pneumonia in $58(6.4 \pm 0.8 \%)$ toxoplasmosis pneumonia in two $(0.2 \pm 0.2 \%)$, and others. All patients were divided into two cohorts: 531 individuals seropositive for $T$. gondii and 376 seronegative ones. It has been found out that general lung pathology is more common in patients with seropositivity to $T$. gondii than in seronegative ones $(43.3 \pm 2.2 \%$ vs. $20.1 \pm 2.0 \%, p<0.001)$. The diagnosis of pneumocystis pneumonia was made ten times more often in the cohort of seropositive patients than in the cohort of seronegative ones $(21.9 \pm 1.8 \%$ vs. $2.1 \pm 0.7 \%$, respectively, $\mathrm{p}<0.001)$ and in deceased patients of these cohorts it was 5.5 times more $(45.1 \pm 5.9 \%$ vs. $8.2 \pm 3.9$, respectively, $\mathrm{p}<0.001)$. In patients with fatal outcome and seropositivity to T. gondii, the incidences of pneumocystis pneumonia increased by $23.2 \%(\mathrm{p}<0.001)$ and bacterial pneumonia by $12.4 \%(\mathrm{p}<0.05)$, whereas in seronegative individuals only pulmonary tuberculosis increased by $13.1 \%(\mathrm{p}<0.05)$ compared with corresponding whole cohorts. Pearson's contingency coefficient showed the mean strength association between infection with $T$. gondii and incidence of pneumocystis pneumonia both in whole cohort $(C=0.272)$ and in patients with fatal outcomes $(C=0.368)$. In conclusion, significantly increasing rate of pneumocystis pneumonia in patients with HIV/AIDS and $T$. gondii infection can be caused by certain synergism between T. gondii and Pneumocystis jirovecii and in some cases overdiagnosis pneumocystis pneumonia due to undiagnosed toxoplasmosis pneumonia.
\end{abstract}

Keywords: toxoplasmosis, pulmonary diseases, immunodeficiency

The parasitic protist Toxoplasma gondii (Nicolle et Manceaux, 1908) (Apicomplexa) is an important opportunistic pathogen. In severly ill immunocompromised patients, the acute hypoxemic respiratory failure (ARF) due to pulmonary infection can be caused by invasive fungal infections (species of Aspergillus and Mucorales, and Pneumocystis jirovecii) and T. gondii infection (Azoulay et al. 2020). The prevalence of $T$. gondii is considerable among population and increased in immunocompromised patients. According to global meta-analysis, the pooled prevalence of $T$. gondii infection in HIV/AIDS patients was $42.1 \%$, compared with $32.0 \%$ in the control (Wang et al. 2017). In University Hospital of South Manchester $100 \%$ of the patients with lung cancer were infected with T. gondii and only $10 \%$ in the controls (Bajnok et al. 2019). Cases of lung lesion caused by $T$. gondii were described in patients with chronic inflammatory arthritis and methotrexate and corticosteroid therapy (Abdulkareem et al. 2017), with leukemia (Scerra et al. 2013), lung cancer (Lu et al.
2015), and also in positive anti-toxoplasma serology bone marrow recipients, who got immunosuppressive therapy for "graft-versus-host" disease after allogenic transplantation, especially in highly endemic for toxoplasma areas (de Medeiros et al. 2001).

Pulmonary toxoplasmosis is a serious pulmonary disease, which has symptoms such as cough, fever, and shortness of breath, rales, myalgias, arthralgias and lymphadenopathy. It can imitate many common lung conditions in the immunocompromised patient, including atypical pneumonia, pneumocystis pneumonia and interstitial lung diseases. Chest X-ray usually reveals bilateral interstitial infiltrates. Lymphadenopathy and hepatosplenomegaly have been reported more frequently in immunocompetent patients than in immunocopromised ones. Serological evidence of active toxoplasmosis arises in immunocompetent but not in immunosuppressed patients. Mortality rate among patients with toxoplasma pneumonia was 55\% (Pomeroy and Filice 1992). In a case report about pulmonary toxoplasmosis

Address for correspondence: Ihor Hnatovych Hryzhak, Department of Infectious Diseases and Epidemiology of Ivano-Frankivsk National Medical University, Halytska Street, 2, Ivano-Frankivsk, 76018, Ukraine. E-mail: ihgryzhak@ukr.net. 
in a patient with methotrexate and corticosteroid therapy a chest computed tomography showed extensive bilateral ground-glass opacities and subcentimetre bilateral hilar lymphadenopathy (Abdulkareem et al. 2017).

In HIV-infected patients, the lung is a major site of $T$. gondii infection after the central nervous system. HIV/ AIDS patients with low CD4 counts $\left(<200\right.$ cells $\left./ \mathrm{mm}^{3}\right)$ have high epidemiological risk as well as immunological risk of toxoplasmosis (Yenilmez and Cetinkaya 2018). Pulmonary toxoplasmosis should be considered in HIV-infected patients with late stage of HIV, CD4 count less than 100 cells $/ \mu 1$ and a poor adherence to Highly Active Antiretroviral Therapy (Velasquez et al. 2016). Disseminated toxoplasmosis with severe necrotising pneumonia with numerous tachyzoites in lung tissue occur in patients with advanced AIDS and toxoplasmosis encephalitis (Pastorello et al. 2018). Also, there is one case report of an AIDS patient with both pulmonary and cerebral toxoplasmosis associated with pneumocystis pneumonia (Rey et al. 2017).

The pulmonary toxoplasmosis rarely occurs in immunocompetent patients. Clinical and image-based findings of toxoplasmosis pneumonia overlap with other kinds of atypical pneumonia. Toxoplasmosis should be considered and immunoglobulin M-specific antibodies should be detected (Leal et al. 2007) in an individual with the specific imaging manifestations such as patchy and flocculent high-density shadows or the ground-glass opacity of the lung. Sometimes immunocompetent individuals acquire toxoplasma pneumonia through inhalation high toxicity species of T. gondii (Shen et al. 2015).

\section{MATERIALS AND METHODS}

\section{Design of the study}

The three-year retrospective cohort research was conducted at the Department of Infectious Diseases and Epidemiology of Ivano-Frankivsk National Medical University and at Ivano-Frankivsk Regional Centre for AIDS Prevention and Fight (Ukraine). It is a fragment of complex research project of Department of Infectious Diseases and Epidemiology entitled "The course of infectious diseases on the background of concomitant pathology, combined chronic infections and invasions, correction of treatment", Ukranian state registration number: 0112 U005012 (2019-2023). The medical data of $907 \mathrm{HIV}$-infected patients (men 58.3\%) with mean age of $35.2 \pm 0.3$ years (18-65 years) within 2013-2015 were studied. According to the test results for IgM, IgG to Toxoplasma gondii, the patients were divided into two groups: 531 seropositive individuals and 376 seronegative ones. Inclusion criteria of the patients to the seropositive cohort were the presence of anti-T.gondii-IgG or IgM, or IgG, or both IgG and IgM in the blood at the first detection. Some previously seronegative individuals were transferred to the seropositive group after the appearance of seroconversion (the appearance of specific IgM or IgG, or both IgG and IgM simultaneously). People who did not have antibodies $T$. gondii at the first detection and without seroconversion during the next 3 years were included in the seronegative cohort.
The diagnosis of pneumocystis pneumonia was based on signs of interstitial pneumonia (X-ray symptom of ground-glass opacity of lung), increased levels of lactate dehydrogenase (LDH) and microscopic identification of Pneumocystis jirovecii in sputum. The PCR test did not use to detect DNA of $P$. jirovecii or T. gondii in a sputum sample.

\section{Statistical analysis}

In both cohorts, we set the percentage of different pulmonary pathologies and their average error $\left(\mathrm{P} \pm \mathrm{m}_{\mathrm{p}}\right)$. Pearson's Chi-square test $(\chi 2)$ was used to determine the significance of differences rates of lung disease in this cohorts. Pearson's contingency coefficient (C) was used to estimate the relation between anti- $T$. gondii seropositive status and each pulmonary disease. The significance of difference between frequency of each pulmonary pathology in group of died patients and in the entire corresponding cohort was assessed by the Student's t-test. Our research was approved by Ethics Commission of Ivano-Frankivsk National Medical University (Expert decision No. 96/17 dated 24 May 2017).

\section{RESULTS}

A total of 531 individuals $(61.4 \%$ men and $38.6 \%$ women) were included in the cohort of patients with seropositivity to Toxoplasma gondii and 376 individuals (53.85\% men and $46.15 \%$ women) were included to the seronegative cohort. In seropositive cohort $91(17.1 \pm 1.6 \%)$ patients had stage I of HIV infection, $101(19.0 \pm 1.7 \%)$ had stage II, $80(15.1 \pm 1.6 \%)$ had stage III, and $259(48.8 \pm 2.2 \%)$ had stage IV. In seronegative cohort $48(12.8 \pm 1.7 \%, \mathrm{p}>0.05)$ patients had stage I of HIV-infection, $78(20.7 \pm 2.1 \%$, p $>0.05)$ had stage II, $76(20.2 \pm 2.1 \%, \mathrm{p}>0.05)$ had stage III, and $174(46.3 \pm 2.6 \%, p>0.05 \%)$ had stage IV. Therefore, the distribution of patients regarding to the stage of HIV-infection was practically the same in both cohorts.

Antiretroviral therapy (ART) was received by 352 patients in the seropositive cohort $(66.3 \pm 2.1 \%)$, which is less than in the cohort of seronegative individuals, i.e., $287(76.3 \pm 2.2 \%, \mathrm{p}=0.002)$. In the seropositive cohort, 134 patients $(25.2 \pm 1.9 \%)$ and 88 ones in the seronegative cohort $(23.4 \pm 2.3 \%, \mathrm{p}>0.05)$ received sulfamethoxazole/ trimethoprim (SMX/TMP) 400/80 mg twice daily for the prevention of pneumocystis pneumonia. In the seropositive cohort 50 persons $(9.4 \pm 1.3 \%)$ and in the seronegative cohort 34 patients $(9.0 \pm 1.5 \%, \mathrm{p}>0.05)$ received azithromycin $(1,200 \mathrm{mg}$ once a week) for the prevention of the infection with the Mycobacterium avium complex. Therefore, SMX/TMP and azithromycin, which can prevent active toxoplasmosis, were administrated to the same proportion of patients in these two cohorts.

During a three-year follow-up of 907 patients opportunistic pulmonary pathology was diagnosed in 306 patients $(33.7 \pm 1.6 \%)$ : pneumocystis pneumonia in 124 $(13.7 \pm 1.1 \%)$, primary pulmonary tuberculosis in 113 $(12.5 \pm 1.1 \%)$, bacterial pneumonia in $58(6.4 \pm 0.8 \%)$, and others were in 10 patients $(1.1 \pm 04 \%)$. Among other diseases there were: toxoplasma pneumonia, pulmonary aspergiloma, Kaposi's sarcoma - two cases of each $(0.2 \pm$ 
Table 1. The distribution of pulmonary pathologies in HIV-infected individuals with and without antibody to Toxoplasma gondii (Nicolle et Manceaux, 1908).

\begin{tabular}{|c|c|c|c|c|c|c|c|}
\hline & \multicolumn{2}{|c|}{$\begin{array}{l}\text { T. gondii-positive } \\
(\mathrm{n}=531)\end{array}$} & \multicolumn{2}{|c|}{$\begin{array}{l}\text { T. gondii-negative } \\
(\mathrm{n}=376)\end{array}$} & \multicolumn{3}{|c|}{ Chi-square test } \\
\hline & Number & $\mathrm{P} \pm \mathrm{m}_{\mathrm{n}}(\%)$ & Number & $\mathrm{P} \pm \mathrm{m}_{\mathrm{n}}(\%)$ & $\chi^{2}$ & $\mathrm{p}$ & $\mathrm{C}$ \\
\hline Pneumocystic pneumonia & $116^{*}$ & $21.9 \pm 1.8$ & 8 & $2.1 \pm 0.7$ & 72.515 & $<0.001$ & 0.272 \\
\hline Bacterial pneumonia & 39 & $7.3 \pm 1.1$ & 19 & $5.1 \pm 1.1$ & 1.931 & 0.165 & 0.046 \\
\hline Pulmonary tuberculosis first detected & 70 & $13.2 \pm 1.5$ & 43 & $11.4 \pm 1.6$ & 0.616 & 0.433 & 0.026 \\
\hline Pulmonary aspergilloma & 1 & $1.2 \pm 0.2$ & 1 & $0.3 \pm 0.3$ & 0.060 & 0.807 & 0.008 \\
\hline Cytomegaloviral pneumonia & 1 & $1.2 \pm 0.2$ & 0 & 0 & 0.709 & 0.400 & 0.028 \\
\hline Bacterial pleurisy & 0 & 0 & 1 & $0.3 \pm 0.3$ & 1.414 & 0.235 & 0.039 \\
\hline Toxoplasmic pneumonia & 2 & $0.4 \pm 0.3$ & 0 & 0 & 1.412 & 0.235 & 0.039 \\
\hline Bronchopulmonary candidiasis & 0 & 0 & 1 & $0.3 \pm 0.3$ & 1,414 & 0.235 & 0.039 \\
\hline Kaposi's sarcoma with lung disseminations & 0 & 0 & 2 & $0.5 \pm 0.4$ & 2.831 & 0.093 & 0.056 \\
\hline Non-Hodgkin's lymphoma of lung & 0 & 0 & 1 & $0.3 \pm 0.3$ & 1.414 & 0.235 & 0.039 \\
\hline Total number of patients with pulmonary pathologies & $230 *$ & $43.3 \pm 2.2$ & 76 & $20.1 \pm 2.0$ & 52.551 & $<0.001$ & 0.234 \\
\hline
\end{tabular}

Table 2. The distribution of pulmonary pathologies in deceased HIV-infected individuals with and without antibody to Toxoplasma gondii (Nicolle et Manceaux, 1908).

\begin{tabular}{|c|c|c|c|c|c|c|c|}
\hline & \multicolumn{2}{|c|}{$\begin{array}{l}\text { T. gondii-positive } \\
\qquad(\mathrm{n}=71)\end{array}$} & \multicolumn{2}{|c|}{$\begin{array}{l}\text { T. gondii-negative } \\
(\mathrm{n}=49)\end{array}$} & \multicolumn{3}{|c|}{ Chi-square test } \\
\hline & Number & $\mathrm{P} \pm \mathrm{m}_{\mathrm{n}} \%$ & Number & $\mathrm{P} \pm \mathrm{m}_{\mathrm{n}} \%$ & $\chi^{2}$ & $\mathrm{p}$ & $\mathrm{C}$ \\
\hline Pneumocystic pneumonia & $32 *$ & $45.1 \pm 5.9$ & 4 & $8.2 \pm 3.9$ & 18.805 & $<0.001$ & 0.368 \\
\hline Bacterial pneumonia & 14 & $19.7 \pm 4.7$ & 7 & $14.3 \pm 5.0$ & 0.593 & 0.442 & 0.070 \\
\hline Pulmonary tuberculosis first detected & 15 & $21.1 \pm 4.8$ & 12 & $24.5 \pm 6.1$ & 0.188 & 0.665 & 0.040 \\
\hline Kaposi's sarcoma with lung disseminations & 0 & 0 & 2 & $4.1 \pm 2.8$ & 2.947 & 0.087 & 0.157 \\
\hline Non-Hodgkin's lymphoma of lung & 0 & 0 & 1 & $2.0 \pm 2.0$ & 1.461 & 0.227 & 0.110 \\
\hline Total number of patients with pulmonary pathology & $61 *$ & $85.9 \pm 4.1$ & 26 & $53.9 \pm 7.1$ & 15.696 & $<0.001$ & 0.386 \\
\hline
\end{tabular}

$0.2 \%$ ); cytomegalovirus pneumonia, pleurisy with bacterial etiology, bronchopulmonary candidiasis, non-Hodgkin's lung lymphoma - one case of each $(0.1 \pm 0.1 \%)$. Using a light microscope, $P$. jirovecii was detected in sputum only in 43 persons $(34.7 \pm 4.3 \%)$ of all 124 patients with the diagnosis of pneumocystis pneumonia. In other cases, this diagnosis established on set of characteristic clinical signs (a ground-glass opacity in the lungs, increasing level of $\mathrm{LDH}$, breathlessness, wheesing in the lungs, fever and a effectiveness of SMX/TMP).

In patients seropositive to $T$. gondii the general lung pathology was twice as frequent as in seronegative ones (43.3 $\pm 2.2 \%$ vs. $20.1 \pm 2.0 \%, \mathrm{p}<0.001$; see Table 1$)$. First of all, the difference concerns pneumocystis pneumonia, the frequency of which in the cohort of seropositive to toxoplasmosis individuals exceeded ten times the frequency in seronegative ones $(21.9 \pm 1.8 \%$ vs. $2.1 \pm 0.7 \%$, respectively, $\mathrm{p}<0.001)$. In this case, the Pearson's contingency coefficient $\mathrm{C}=0.272$ reflected the mean strength association between $T$. gondii infection and pneumocystis pneumonia. It is noteworthy that Kaposi's sarcoma with lung dissemination was observed only in patients with seronegative status to $T$. gondii - two cases $(0.5 \pm 0.4 \%)$. In seropositive individuals Kaposi's sarcoma occurred only as cutaneous manifestations $-2(0.4 \pm 0.3 \%)$.

Within three years, 120 patients died: 71 patients $(13.4$ $\pm 1.5 \%)$ of the seropositive cohort and 49 patients $(13.0 \pm$ $1.7 \%$ ) of the seronegative one. The mortality rate did not differ in the two cohorts $(p>0.05)$. Among persons with fatal outcome, in seropositive individuals the total number of patients with general lung pathology was higher than in seronegatives ones $(85.9 \pm 4.1 \%$ vs. $53.9 \pm 7.1 \%, \mathrm{p}<$ 0.001 ; see Table 2). In seropositive deceased persons, the pneumocystis pneumonia frequency also was higher 5.5 times than in seronegative ones $(45.1 \pm 5.9 \%$ vs. $8.2 \pm$ $3.9 \%, \mathrm{p}<0.001)$, and it had a mean strength association with infection with $T$. gondii $(\mathrm{C}=0.368$; see Table 2$)$.

In deceased seropositive to toxoplasmosis individuals, the frequency of general lung pathology was significantly higher by $42.6 \%$ compared with this entire cohort $(85.9 \pm$ $4.1 \%$ vs. $43.3 \pm 2.2 \%$, respectively, $t=9.15, \mathrm{p}<0.001$ ), including increased incidences of pneumocystis pneumonia by $23.2 \%(45.1 \pm 5.9 \%$ vs. $21.9 \pm 1.8 \%$, respectively, $\mathrm{t}=3.76$, $\mathrm{p}<0.001)$ and bacterial pneumonia by $12.4 \%(19.7 \pm 4.7 \%$ vs. $7.3 \pm 1.1 \%$, respectively, $t=2.55, \mathrm{p}<0.05$; Fig. 1$)$. In the group of deceased persons out of the seronegative cohort, the frequency of general lung pathology also increased by $33.7 \%$ compared with this entire cohort $(53.9 \pm 7.1 \%$ vs. $20.2 \pm 2.1 \%, \mathrm{t}=4.56, \mathrm{p}<0.001)$, mainly due to increasing incidence of pulmonary tuberculosis by $13.1 \%(24.5 \pm$ $6.1 \%$ vs. $11.4 \pm 1.6 \%, \mathrm{t}=2.05, \mathrm{p}<0.05$; Fig. 1$)$. Thus, difference was observed between deceased persons compared with their entire cohorts: the incidence of pneumocystis and bacterial pneumonia increased in seropositive individuals but pulmonary tuberculosis increased only in seronegative ones.

Not all of these pulmonary diseases were the main causes of death in patients, but they were observed during the follow-up, and reflected a gradual worsening of patients' health condition. In deceased seropositive patients, pulmonary pathology as a cause of death was 


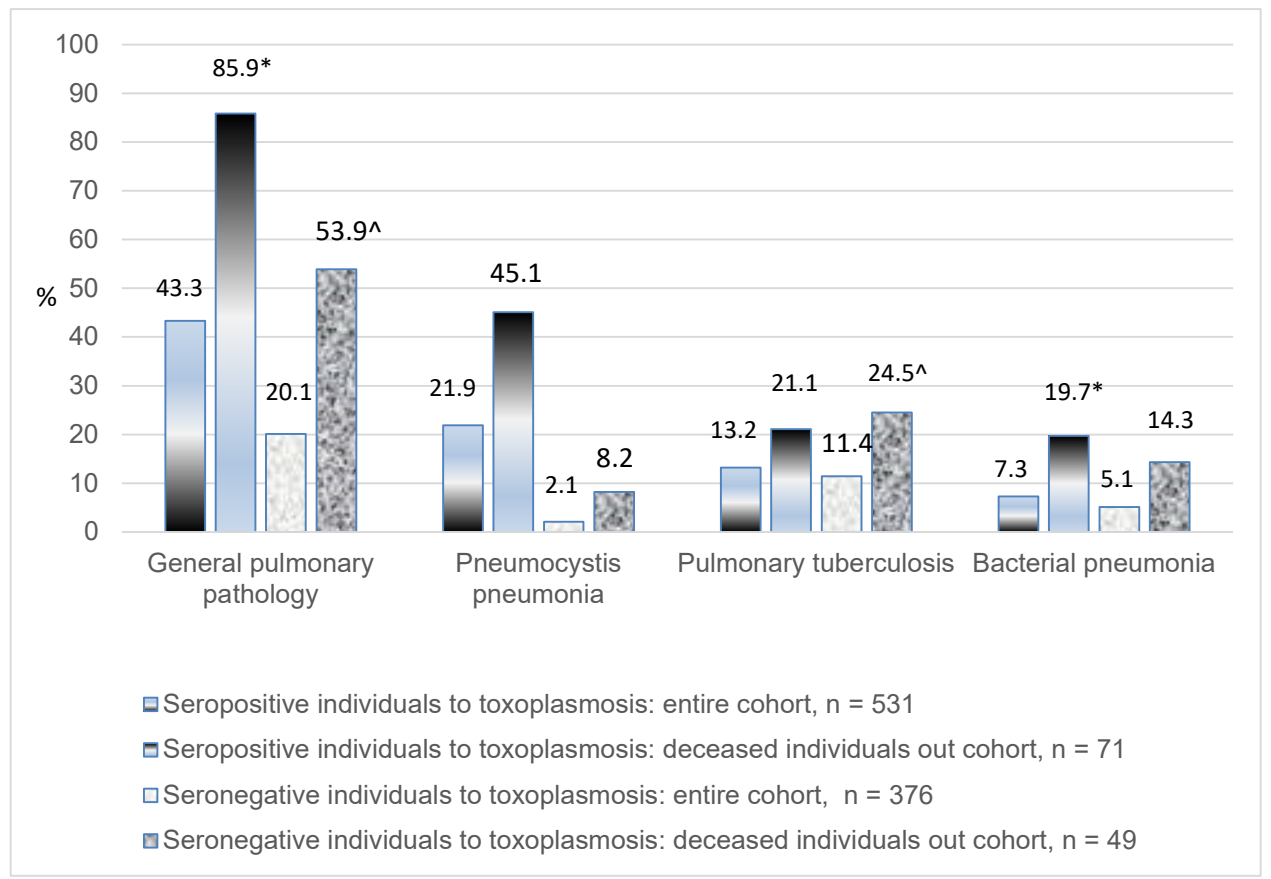

Fig. 1. Frequency of different pulmonary diseases in groups of deceased patients out of seropositive and seronegative cohorts to Toxoplasma gondii (Nicolle et Manceaux, 1908). * - the difference between the indices in the entire cohort of seropositive patients and in the group of deceased ones out of this cohort with $\mathrm{p}<0.05 ;^{\wedge}-$ the difference between the indices in the entire cohort of seronegative patients and in group of deceased ones out this cohort with $\mathrm{p}<0.05$.

presented as the following pathologies: tuberculosis -12 patients $(16.9 \pm 4.5 \%)$, pneumocystis pneumonia $-1(1.4 \pm$ $1.4 \%)$, bacterial destruction $-1(1.4 \pm 1.4 \%)$; in the group of seronegative deceased persons: tuberculosis - in 11 patients $(2.5 \pm 6.0 \%)$, Kaposi's sarcoma $-2(4.1 \pm 2.8 \%)$, non-Hodgkin's lymphoma - $1(2.0 \pm 2.0 \%)$, pneumocystis pneumonia $-1(2.0 \pm 2.0 \%)$.

\section{DISCUSSION}

It has been found out that general pulmonary pathology occurs more often in individuals seropositive to Toxoplasma gondii than in seronegatives ones, which may be explained by some influence of $T$. gondii on the development of pulmonary pathology. In particular, it was found that the frequency of pneumocystis pneumonia in the cohort of seropositive individuals is ten times higher than in seronegative one. In many cases, Pneumocystis jirovecii was not detected in sputum, so doctors made a diagnosis based on clinical symptoms that are common to both pneumocystis pneumonia and toxoplasmosis pneumonia. Moreover, treatment with SMX/TMP is effective against both pathogens. Therefore, toxoplasmosis pneumonia in some cases could be hidden under the mask of overdiagnosed pneumocystis pneumonia.

In patients with fatal HIV-infection, the incidence of general pulmonary pathology increased significantly in both groups during the follow-up, reflecting deterioration in general health condition and decreased immunity. In deceased seropositive persons, the most common lung pathology was pneumocystis pneumonia. Instead, pulmonary tuberculosis was the most common in the seronegative persons. This may be due to pneumocystis pneumonia as first manifestation in seropositive patients and tuberculosis in seronegative ones. When there is manifestation of pneumocystis pneumonia, all patients receive isoniazid for the tuberculosis prevention, and when pulmonary tuberculosis manifests, patients receive SMX/TMP for the pneumocystis pneumonia prevention. Also, Pearson's contingency coefficient $(\mathrm{C}=0.368)$ shows a medium strength relationship between toxoplasmosis infection and pneumocystis pneumonia and suggests some association between pneumocystosis and toxoplasmosis. These data indicate a possible synergism between $T$. gondii and $P$. jirovecii.

Therefore, under conditions of immunodeficiency, the body protection against pathogenic fungi depends on innate immunity, namely on the function of alveolar macrophages. Normally, IFN $\gamma$ reprogrammed macrophages to a permissive M1-biased phenotype. M1 cells exhibited significantly increased production of iNOS, NO, TNF- $\alpha$, IL- $1 \beta$, and IL-6 (Bhagwat et al. 2018). However, ToxoROP16 induced macrophage bias to $\mathrm{M} 2$ cells (in vitro), showing decreased production of NO and iNOS and increased expression of Arg-1, IL-10 and TGF- $\beta 1$ (Denkers 2003). In turn, IL-10 is a potent antagonist of IFN-gamma, which is a critical cytokine involved in mediating $T$. gondii-induced immunosuppression in the infected host (Khan et al. 1995). Also, intracellular $T$. gondii infection causes a blockade in the NFkappaB macrophage signaling pathway and reduced IL-12 and TNF-alpha production. The parasite also prevents STAT1 activity, resulting in decreased levels of IFN-gamma-stimulated MHC surface antigen expression. Extracellular pathways of suppression involve soluble mediators such as IL-10 and lipoxins that have potent IL-12 down-regulatory effects (Meira et al. 2014). For this reason, T. gondii can prevent the eradica- 
tion of pneumocystis and bacterial pathogens from the lungs and help pneumonia development.

Some lung oncology problems such as pulmonary dissemination of Kaposi's sarcoma and non-Hodgkin's lymphoma were detected only in seronegative deceased persons, which remained without satisfactory explanation. It can be explained by profound immunodeficiency in some patients with seronegative status who did not take ART for a long time. These findings are important for the physician as they indicate the necessity of the diagnosis and treatment of active T. gondii infection in HIV-positive people to prevent not only toxoplasmosis lung lesions but could play certain role in prevention pneumocystis pneumonia.

The results of the study may be affected by different number of patients in the two groups who used ART. Thus, the number of seropositive individuals to $T$. gondii who used ART was by $10 \%$ lower than seronegative ones. It has not been known how many people had the compliance to ART and how many of them violated the treatment regimen. In the mentioned period of the study the ART was prescribed for patients with advanced stages of AIDS, so satisfactory reconstitution of immunity in many patients was not observed. This could lead to a worsening of immunodeficiency and development of various opportunistic diseases including pneumocystis pneumonia. This pathological process does not depend on the presence of $T$. gondii.

In conclusion, significantly increasing rate of pneumocystis pneumonia in patients with HIV/AIDS and T. gondii infection can be caused by certain synergism between $T$. gondii and $P$. jirovecii and some cases of overdiagnosis pneumocystis pneumonia due to undiagnosed toxoplasmosis pneumonia.

Acknowledgements. We would like to thank the doctors, nurses and other medical staff of the Ivano-Frankivsk Regional Centre for AIDS Prevention and Fight for their professional assistance through-out the study.

\section{REFERENCES}

Abdulkareem A., D’Souza R.S., Patel N., Donato A. 2017: A rare case of pulmonary toxoplasmosis in a patient with undifferentiated inflammatory arthritis on chronic methotrexate and corticosteroid therapy. BMJ Case Rep. 2017: bcr2017221252.

Azoulay E., Russell L., Van de Louw A., Metaxa V., Bauer Ph., Povoa P., Montero J.G., Loeches I.M., Mehta S., Puxty K., Schellongowski P., Rello J., Mokart D., Lemiale V. Mirouse A. 2020: Diagnosis of severe respiratory infections in immunocompromised patients. Intensive Care Med. 46: 298-314.

Bajnok J., Tarabulsi M., Carlin H., Bown K., Southworth T., Dungwa J., Singh D., Lun Zh., Smyth L., Hide G. 2019: High frequency of infection of lung cancer patients with the parasite Toxoplasma gondii. ERJ Open Res. 5: 00143-2018.

Bhagwat S.P., Gigliotti F., Wang J., Wang Z., Notter R.H., Murphy P.S., Rivera-Escalera F., Malone J., Jordan M.B., Elliott M.R., Wright T.W. 2018: Intrinsic programming of alveolar macrophages for protective antifungal innate immunity against Pneumocystis infection. Front. Immunol. 9: 2131.

DenKers E.Y. 2003: From cells to signaling cascades: manipulation of innate immunity by Toxoplasma gondii. FEMS Immunol. Med. Microbiol. 39: 193-203.

Khan I.A., Matsuura T., Kasper L.H. 1995: IL-10 mediates immunosuppression following primary infection with Toxoplasma gondii in mice. Parasite Immunol. 17: 185-195.

Leal F.E., Cavazzana C.L., Andrade H.F., Galisteo A.J., Mendonca J.S., Kallas E.G. 2007: Toxoplasma gondii pneumonia in immunocompetent subjects: case report and review. Clin. Infect. Dis. 44: e62-66.

Lu N., Liu C., Wang J., Ding Y., Ai Q. 2015: Toxoplasmosis complicating lung cancer: a case report. Int. Med. Case Rep. J. 8: $37-40$.

de Medeiros B.C., de Medeiros C.R., Werner B., Loddo G., Pasquini R., Bleggi-Torres L.F. 2001: Disseminated toxoplasmosis after bone marrow transplantation: report of 9 cases. Transpl. Infect. Dis. 3: 24-28.
Meira C.S., Pereira-Chioccola V.L., Vidal J.E., de Mattos C.C., Motoie G., Costa-Silva T.A., Gava R., Frederico F.B., De Mattos L.C. 2014: Toxoplasma groups. Cerebral and ocular toxoplasmosis related with IFN- $\gamma$, TNF- $\alpha$, and IL-10 levels. Front. Microbiol. 5: 492.

Pastorello R.G., Costa A.C., Sawamura M.V., Nicodemo A.C., Duarte-Neto A.N. 2018: Disseminated toxoplasmosis in a patient with advanced acquired immunodeficiency syndrome. Autops. Case Rep. 8: e2018012.

Pomeroy C., Filice G.A. 1992: Pulmonary toxoplasmosis: a review. Clin. Infect. Dis. 14: 863-870.

Rey M.F., Mary C., Sanguinetti D., Ranque S., Bartoli C., L'OlLivier C. 2017: Successful treatment of pulmonary and cerebral toxoplasmosis associated with pneumocystis pneumonia in an HIV patient. Diseases 5: 35.

Scerra S., Coignard-Biehler H., Lanternier F., Suarez F., Charlier-Woerther C., Bougnoux M-E., Gilquin J., Lecuit M., Hermine O., Lortholary O. 2013: Disseminated toxoplasmosis in non-allografted patients with hematologic malignancies: report of two cases and literature review. Eur. J. Clin. Microbiol. Infect. Dis. 32: 1259-1268.

Shen C., Zhang G., Song X., Xie S., Wang Ch. 2015: Toxoplasma gondii pneumonia in an immunocompetent individual. Am. J. Med. Sci. 350: 70-71.

Velasquez J.N., Ledesma B.A., Nigro M.G., Vittar N., Rueda N., De Carolis L., Figueiras O., Carnevale S., Corti M. 2016: Pulmonary toxoplasmosis in human immunodeficiency virus-infected patients in the era of antiretroviral therapy. Lung India 33: 88-91.

Yenilmez E., Cetinkaya R.A. 2019: Difference in Toxoplasma gondii seroprevalence rates due to low and high CD4 counts in patients with HIV/AIDS. Turkiye Parasitol. Derg. 43: 1-7.

WANG Z.-D., LiU H.-H., Ma Z.-X., Ma H.-Y., Li Z.-Y., YANG Z.B., Zhu X.-Q., Xu B., Wei F., Liu Q. 2017. Toxoplasma gondii infection in immunocompromised patients: a systematic review and meta-analysis. Front. Microbiol. 8: 389.

Cite this article as: Hryzhak I.H. 2021: Invasion with Toxoplasma gondii can promote pneumocystis pneumonia in individuals with HIV/AIDS. Folia Parasitol. 68: 018. 